\title{
Cloning, sequence analysis, and expression of cDNA coding for the major house dust mite allergen, Der $\mathrm{f} 1$, in Escherichia coli
}

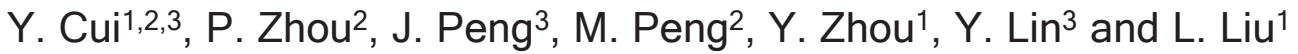 \\ ${ }^{1}$ Yancheng Health Vocational and Technical College, Yancheng, Jiangsu Province, the People's Republic \\ of China \\ ${ }^{2}$ State Key Laboratory of Tropical Crop Biotechnology, Institute of Tropical Science, China Academy of \\ Tropical Agricultural Science, Haikou, the People's Republic of China \\ ${ }^{3}$ Hainan Medical College, Haikou, the People's Republic of China
}

Correspondence to: P. Zhou, State Key Laboratory of Tropical Crop Biotechnology, Institute of Tropical

Science, China Academy of Tropical Agricultural Science, Chengxi Road, Haikou 571101, the People's

Republic of China

E-mail:6801609@163.com

\begin{abstract}
Our objective was to clone, express and characterize adult Dermatophagoides farinae group 1 (Der $f$ 1) allergens to further produce recombinant allergens for future clinical applications in order to eliminate side reactions from crude extracts of mites. Based on GenBank data, we designed primers and amplified the cDNA fragment coding for Der $f$ by nested-PCR. After purification and recovery, the cDNA fragment was cloned into the pMD19-T vector. The fragment was then sequenced, subcloned into the plasmid pET28a(+), expressed in Escherichia coli BL21 and identified by Western blotting. The cDNA coding for Der $f 1$ was cloned, sequenced and expressed successfully. Sequence analysis showed the presence of an open reading frame containing $966 \mathrm{bp}$ that encodes a protein of 321 amino acids. Interestingly, homology analysis showed that the Der $\mathrm{p} 1$ shared more than $87 \%$ identity in amino acid sequence with Eur $\mathrm{m} 1$ but only $80 \%$ with Der $\mathrm{f} 1$. Furthermore, phylogenetic analyses suggested that $D$. pteronyssinus was evolutionarily closer to Euroglyphus maynei than to $D$. farinae, even though $D$. pteronyssinus and $D$. farinae belong to the same Dermatophagoides genus. A total of three cysteine peptidase active sites were found in the predicted amino acid sequence, including 127-138 (QGGCGSCWAFSG), 267-277 (NYHAVNIVGYG) and 284-303 (YWIVRNSWDTTWGDSGYGYF). Moreover, secondary structure analysis revealed that Der $f 1$ contained an $\alpha$ helix (33.96\%), an extended strand (17.13\%), a ß turn (5.61\%), and a random coil (43.30\%). A simple three-dimensional model of this protein was constructed using a Swiss-model server. The cDNA coding for Der $f 1$ was cloned, sequenced and expressed successfully. Alignment and phylogenetic analysis suggests that $D$. pteronyssinus is evolutionarily more similar to $E$. maynei than to $D$. farinae.
\end{abstract}

Key words: Dermatophagoides farinae; Der f 1; Recombinant allergens; Cloning; Expression; Bioinformatics

Research supported by the National Sciences Foundation of China (\#30060166), Natural Science Fund of Hainan Province (\#2005-80556), and Hainan Provincial Health Department (\#2005-6).

Received September 28, 2007. Accepted April 23, 2008

\section{Introduction}

It is well known that house dust mites are associated with IgE-mediated allergies such as asthma, rhinitis, conjunctivitis, urticaria, and atopic dermatitis. Although aller- gens from several mite families have been reported, including Acaridae, Glycophagidae and Pyroglyphidae, proteins produced by mites of the Pyroglyphidae family, especially the species Dermatophagoides farinae, D. pteronyssinus, and Euroglyphus maynei are the most important 
mites because they affect human beings. Indeed, these species are commonly found in human dwellings worldwide (1). As complex organisms, house dust mites produce thousands of different proteins and macromolecules, and over $30 \mathrm{lgE}$-binding components have been demonstrated in patients allergic to house dust mites by immunological analysis (2). Of these allergens, group 1 is the major one and has been identified to be homologous with cysteine proteases (3).

Although the recombinant allergen Der $p 1$ has been studied in clinical trials in some developed countries, currently, only the crude extracts from $D$. farinae are used in diagnosis and immunology in China. To characterize house dust mite allergens, we cloned, sequenced and analyzed the cDNA encoding the major allergen $D$. farinae group 1 (Der $f$ 1). Interestingly, results of homology searches and phylogenetic analyses suggested that $D$. farinae seemed to be more similar to $E$. maynei than to $D$. pteronyssinus, even though $D$. farinae and $D$. pteronyssinus belong to the same Dermatophagoides genus. Here, we aimed to clone and sequence Der $\mathrm{f} 1$ and describe its relevant molecular characteristics.

\section{Material and Methods}

\section{$D$. farinae culture and isolation of adult mites}

To isolate house dust mites, we first obtained house dusts from the floors of rice and flour shops in Haikou City, Hainan Island, and isolated house dust mites under a stereomicroscope. All of the mites potentially belonging to $D$. farinae were placed in a culture chamber. After about 2 months, the whole culture in the chamber was examined under a stereomicroscope to select for $D$. farinae. Subsequently, mites considered to be $D$. farinae were cultivated in small culture chambers for pure culture. Two months later, the mites were taken from the chambers to identify mite species. If the mite was confirmed to be $D$. farinae, all of the mites in the chamber were considered to be $D$. farinae. These mites were then raised on a large-scale basis at $25^{\circ} \mathrm{C}$ and $75 \%$ relative humidity. A culture medium consisting of yeast, wheat flour and rice was used for the large-scale cultivation. According to our previous protocols (4), the pure adults were isolated as follows: the whole culture was placed on glass plates, and after 30 min the culture media were removed manually. The adult mites, larval mites and some media were collected into a small ceramic cup using a small writing brush. Under a lamp, the adults moved rapidly to the basal part of the ceramic cup. The adults were removed with a writing brush under a microscope, and about 600 mites were chosen for total RNA isolation.

\section{Isolation of total RNA}

Total RNA was isolated from mites using an RNA isolator (Code \#D312; TaKaRa Biotechnology Limited Company, Dalian, China) according to manufacturer instructions. Briefly, about 600 live adult mites were rapidly frozen in liquid nitrogen and $1 \mathrm{~mL}$ RNA isolator was added. The mites in RNA isolator were homogenized using a PowerGen 125 Tissue Homogenizer (Fisher Scientific, UK) starting at 5000 rpm and gradually increasing to approximately 20,000 rpm for 30-60 s at room temperature. After homogenization, the entire content of the tissue homogenizer was transferred to an Eppendorf tube and $0.2 \mathrm{~mL}$ chloroform was added. After precipitation and air-drying, the RNA pellet was dissolved in RNase-free water and stored at $-80^{\circ} \mathrm{C}$.

\section{Polymerase chain reaction}

The oligonucleotide primers $F$ ( 5 'GGATCCATGAAATT CGTTTTGGCCATTG3'), R0 (5'TCGCAAGAGTAGTTGTTT TTAT3') and R (5'CTCGAGTCACATGATTACAACATAT GGATATT3') were designed on the basis of the sequence of the Der $f 1$ gene (GenBank AB034946) and synthesized. To facilitate cloning, the $\mathrm{F}$ and $\mathrm{R}$ primers contained a $\mathrm{BamHI}$ site at the $5^{\prime}$ end of the coding sequence (underlined) of $\mathrm{F}$ and an Xhol site at the $5^{\prime}$ end of $\mathrm{R}$, respectively. First, a reverse transcriptase-polymerase chain reaction (RT-PCR) amplification was performed using the total RNA isolated from the mites with the one-step RNA PCR kit (Code \#DRR024A, TaKaRa) according to manufacturer instructions. In this reaction, the final concentrations of the individual components per reaction ( $50 \mu \mathrm{L}$ final volume) were as follows: 20 pmol of the primers $F$ and $R 0$ each ( $1 \mu \mathrm{L}$ each), total RNA $(4 \mu \mathrm{L}), 2 \mathrm{X}$ one-step RNA PCR buffer $(25 \mu \mathrm{L}), 2.5$ $\mathrm{mM}$ of the dNTP mixture $(2 \mu \mathrm{L}), 40 \mathrm{U} / \mu \mathrm{L}$ of an RNase inhibitor $(1 \mu \mathrm{L}), 5 \mathrm{U} / \mu \mathrm{L}$ of the M-MLV reverse transcriptase $\mathrm{XL}(0.5 \mu \mathrm{L})$, and $5 \mathrm{U} / \mu \mathrm{L}$ Ex Taq $(1 \mu \mathrm{L})$. RT-PCR was performed in the PCR Thermal Cycler Dice (Code \#TP600, TaKaRa). The PCR conditions used in this study included an initial incubation for 2 min at $94^{\circ} \mathrm{C}$ followed by 30 cycles of 15 $\mathrm{s}$ at $98^{\circ} \mathrm{C}, 30 \mathrm{~s}$ at $57^{\circ} \mathrm{C}$, and $1 \mathrm{~min}$ at $72^{\circ} \mathrm{C}$. After a final incubation for $5 \mathrm{~min}$ at $72^{\circ} \mathrm{C}$, the amplicons were analyzed by agarose gel electrophoresis (1.0\%) and visualized with ImageMaster ${ }^{\circledR}$ VDS (USA). To obtain the gene fragment encoding Der $f 1$, the above amplicon was treated as a template and the $F$ and $R$ primers were used in the second PCR with the PrimeSTARTM HS DNA polymerase kit (Code \#DR010A, TaKaRa). In this reaction, the final concentrations of the components per reaction ( $50 \mu \mathrm{L}$ final volume) were as follows: 20 pmol each of the $F$ and R primers $(0.5 \mu \mathrm{L}$ each), the above amplicons $(1 \mu \mathrm{L}), 2 \mathrm{X}$ PrimerSTARTM buffer $(25 \mu \mathrm{L}), 2.5 \mathrm{mM}$ of the dNTP mixture $(2 \mu \mathrm{L})$, and $2.5 \mathrm{U} /$ $\mu \mathrm{L}$ of the PrimeSTARTM HS DNA polymerase $(0.5 \mu \mathrm{L})$. PCR 
was performed in the same PCR Thermal Cycler Dice as described above with an initial incubation of $5 \mathrm{~min}$ at $94^{\circ} \mathrm{C}$, followed by 30 cycles of $10 \mathrm{~s}$ at $98^{\circ} \mathrm{C}, 10 \mathrm{~s}$ at $57^{\circ} \mathrm{C}$, and $1 \mathrm{~min}$ at $72^{\circ} \mathrm{C}$. The $P C R$ product was then analyzed and visualized as described above.

\section{Cloning and DNA sequencing}

After being recovered from the gel using the agarose gel DNA purification kit ver. 2.0 (Code \#DV805, TaKaRa), a poly "A" tail was added to the PCR-amplified DNA with the DNA A-tailing kit (Code \#D404, TaKaRa) and ligated into the pMD19-T simple vector (Code \#D104, TaKaRa). The Escherichia coli JM109 (Code \#D9052, TaKaRa) was then transformed with the recombinant plasmids and positive clones were selected by blue/white screening on LuriaBertani (LB) plates containing $100 \mu \mathrm{g} / \mathrm{mL}$ ampicillin, and confirmed by restriction enzyme analysis and automatic DNA sequencing.

\section{Construction of expression plasmids}

The recombinant plasmids constructed previously were digested with BamHI and $\mathrm{Xhol}$ to release the Der $\mathrm{f} 1$ fragment. After recovery from the gel using the agarose gel DNA purification kit ver. 2.0 (Code \#DV805, TaKaRa), the fragment was subcloned into expression vector pET28a(+) (kit lot \#N72770, Novagen, Germany) to create pET28a(+)Der $f 1$ using the DNA ligation kit (Code \#D6023, TaKaRa). E. coli competent JM109 cells (Code \#D9052, TaKaRa) were transformed with $\mathrm{pET} 28 \mathrm{a}(+)$-Der $\mathrm{f} 1$ plasmids and positive clones were selected by blue/white screening and determined by restriction enzyme analysis with BamHI and Xhol.

\section{Expression of rDer $\mathrm{f} 1$ in $E$. coli BL21}

Five microliters of $\mathrm{pET} 28 \mathrm{a}(+)$-Der $\mathrm{f} 1$ plasmid purified with the MiniBEST plasmid purification kit ver. 2.0 was used to transform $100 \mu \mathrm{L}$ E. coli BL21 (Stratagene, USA). The $E$. coli BL21 carrying pET28a(+)-Der $\mathrm{f} 1$ was grown on LB plates containing $50 \mu \mathrm{g} / \mathrm{mL}$ kanamycin at $37^{\circ} \mathrm{C}$ overnight. The cells were further cultured for $2 \mathrm{~h}$ in $2 \mathrm{~mL}$ LB solution containing $1 \mathrm{mM}$ isopropyl-ß-D-thiogalactopyranoside at $37^{\circ} \mathrm{C}$ to induce the tac promoter. The E. coli cells were harvested by centrifugation and $20 \mu \mathrm{L}$ of the cell pellet including both lysed supernatant and the whole cells was taken and $20 \mu \mathrm{L}$ of $2 X$ SDS sample buffer was added to the mixture. The samples were heated for $10 \mathrm{~min}$ at $95^{\circ} \mathrm{C}$ and subjected to SDS-PAGE.

\section{Western blotting}

For Western blotting, proteins were transferred to a PVDF membrane (Tiangen, Biotech Co. Led, Beijing, China), the blot was incubated with the primary antibody of the HisTag Mab at $4^{\circ} \mathrm{C}$ overnight, followed by incubation with the secondary anti-mouse goat IgG antibody AP conjugated for $1 \mathrm{~h}$ at room temperature, and proteins were then visualized using $3 \mathrm{~mL}$ BCIP/NBT (Roche, Switzerland).

Nucleotide sequence, inferred amino acid sequence, alignment, and phylogeny

Sequences were edited to remove the vector sequence and the extra restriction sites. One open reading frame (ORF) was obtained using the ORF finder at the National Center for Biotechnology Information (NCBI) website. The amino acid sequence of Der $f 1$ was deduced using the Translate Tools in the ExPaSy web server, and matched with the group 1 allergen and cysteine protease sequences published in GenBank using BLAST. Next, the published sequences of ticks and mites were chosen for alignment using VECTOR NTI 9.0 software (IBI, New Haven, CT, USA), and a polygenic tree was constructed using the PAUP 4.0b10 software.

Secondary and three-dimensional structure analysis

The secondary structure prediction was performed by SOPMA, and a simple three-dimensional model was constructed with a template (PDB ID:1XKG) using the SWISSMODEL server.

\section{Results}

\section{Cloning and sequence of Der $\mathrm{f} 1 \mathrm{cDNA}$}

Total RNA was isolated from adult mites and Der $\mathrm{f} 1$ cDNA fragments were amplified using RNA isolated from adult mites by nested-PCR (966 bp, Figure 1). The recovered PCR product was cloned into the plasmid vector pMD19-T, which was used to transform competent $E$. coli cells (JM109). The positive clones were selected by blue/ white screening, determined by restriction enzyme digestion and sequenced to confirm their identity by automatic DNA sequencing (Figure 2, the vector sequence and the added restriction sites were removed from the sequencing results). The sequencing results demonstrated that our Der $f 1$ cDNA is identical to the published sequence (GenBank AB034946), except for a point mutation at position $23(\mathrm{C}>\mathrm{T})$. Using the ORF finder in the NCBI web server, a complete ORF was found within the Der $f 1 \mathrm{cDNA}$, whose length was $966 \mathrm{bp}$ from the start codon ATG to the stop codon TGA (Figure 2).

Inferred amino acid sequence and its physicochemical property

In order to forecast the physicochemical property of the 
Figure 1. Amplification of Der $\mathrm{f} 1$ by nestedPCR. Total RNA was isolated from mites using an RNA isolator and subjected to RTPCR as described in Material and Methods. The PCR products were separated on $1 \%$ agarose gel containing ethidium bromide. Lane 1, DNA marker DL2,000; lane 2, PCR product.

Figure 2. The amino acid sequence of Der $f$ 1 deduced from its nucleotide sequence using Translate Tools (http://www.expasy. org). The bold numbers 1-18 represent the signal peptide sequence, the underlined amino acids 19-98 represent the sequence coding for pro-peptides.

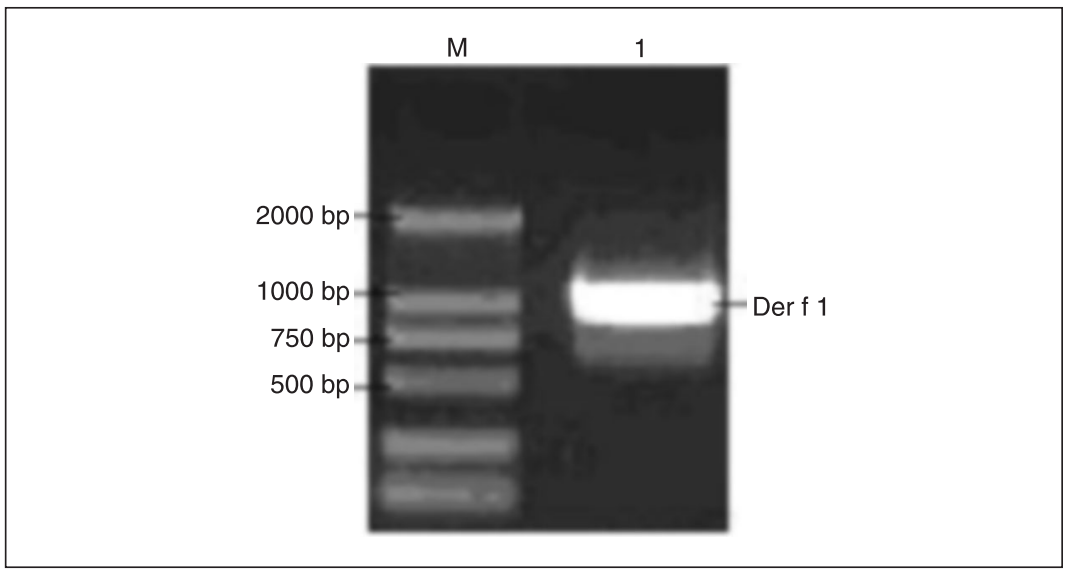

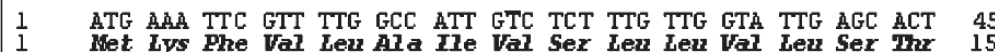
46 GTT TAT GCT CGT CCA GCT TCA ATC AAA ACT TTT GAA GAA TTC AAA 90 16 Val Tyr Ala Arg Pro Ala Ser Ile Lys Thr Phe Glu Glu Phe Lys 30

91 AAA GCC TTC AAC AAA AAC TAT GCC ACC GTT GAA GAG GAA GAA GTT 135 31 Lys Ala Phe Asn Lys Asn Tyr Ala Thr Val Glu Glu Glu Glu Val 45

136 GCC CGT AAB AAC TTT TTG GAB TCA TTG AAA TAT GTT GAA GCT AAC 46 Ala Arg Lys Asn Phe Leu Glu Ser Leu Lys Tyr Val Glu Ala Asn

181 AABA GGT GCC ATC AAC CAT TTG TCC GAT TTG TCA TTG GAT GAA TTC 61 LYS Gly Ala Ile Asn His Leu Ser Asp Leu Ser Leu Asp Glu Phe

226 AAA AAC CGT TAT TTG ATG AGT GCT GAA GCT TTT GAM CAA CTC AAA

76 Lys Asn Arg Tyr Leu Met Ser Ala Glu Ala Phe Glu Gln Leu LYs

271 ACT CAA TTC GAT TTG AAT GCC GAA ACA AGC GCT TGC CGT ATC AAT

91 Thr Gln Phe Asp Leu Asn Ala Glu Thr ser Ala CYs Arg Ile Asn

316 TCG GTT AAC GTT CCA TCG GAA TTG GAT TTA CGA TCA CTG CGA ACT

106 Ser Val Asn Val Pro Ser Glu Leu Asp Leu Arg Ser Leu Arg Thr

361

121

406

136

GTC ACT CCA ATC CGT ATG CAA GGA GGC TGT GGT TCA TGT TGG GCT Val Thr Pro Ile Arg Met Gln Gly Gly Cys Gly ser Cys Trp Ala

TTC TCT GGT GTC GCC GCA ACT GÄA TCA GCT TÄT TTG GCC TAC CGT Phe ser Gly Val Ala Ala Thr Glu Ser Ala Tyr Leu Ala Tyr Arg

451 ÄC ACG TCT TTG GAT CTT TCT GÄ CAG GÄ CTC GTC GAT TGC GCA AAC ACG TCT TTG GAT CTT TCT GÄ CAG GAA CTC GTC GAT TGC GCA

TCT CAA CAC GGA TGT CAC GGC GAT ACA ATA CCA AGA GGC ATC GAA ser Gln His GlY CYs His GlY Asp Thr Ile Pro Arg GlY Ile Glu TAC ATC CAB CAM AAT GGT GTC GTT GAB GAM AGA AGC TAT CCA TAC GTT GCA CGA GAA CAA CAA TGC CGA CGA CCA AAT TCG CAA CAT TAC Val Ala Arg Glu Gln Gln Cys Arg Arg Pro Asn ser Gln His Tyr GGT ATC TCA AAC TAC TGC CAA ATT TAT CCA CCA GAT GTG AAA CAÁ Gly Ile ser Asn Tyr Cys Gln Ile Tyr Pro Pro Asp Val Lys Gln ATC CGT GAA GCT TTG ACT CAA ACA CAC ACA GCT ATT GCC GTC ATT Ile Arg Glu Ala Leu Thr Gln Thr His Thr Ala Ile Ala Val Ile ATT GGC ATT AAA GAT TTG AGA GCT TTT CAA CAT TAT GAT GGA CGA Ile Gly Ile Lys Asp Leu Arg Ala phe Gin His Tyr Asp Gly Arg ACA ATC ATT CAA CAT GAC AAT GGT TATT CAA CCA AAC TAT CAT GCC Thr Ile Ile Gln His Asp Asn Gly Tyr Gln Pro Asn TYr His Ala GTC AAC ATT GTC GGT TAC GGÁ AGT ACA CẢ̆ GGC GTC GAT TAT TGG Val Asn Ile val Gly Tyr Gly ser Thr Gln Gly Val Asp Tyr Trp ATC GTA CGA AAC AGT TGG GAT ACT ACC TGG GGT GAT AGC GGA TAC Ile Val Arg Asn Ser Trp Asp Thr Thr Trp GlY Asp Ser GlY TYr 300 GGA TAT TTC CAA GCC GGA AAC AAC CTC ATG ATG ATC GAA CAA TAT 945 Gly Tyr Phe Gln Ala Gly Asn Asn Leu Met Met Ile Glu Gln Tyr 315 CCA TAT GTT GTA ATC ATG TGA 966 Pro Tyr Val Val Ile Met End 
Der $\mathrm{f} 1$ protein, the amino acid sequence of Der $\mathrm{f} 1$ was deduced from its nucleotide sequence using the Translate Tools software in the ExPaSy web server (Figure 2). Based on ProtParam Tools, the putative protein had a molecular mass of $36,419 \mathrm{kDa}$ and a theoretical pl of 5.66 , the most likely cleavage site of which is between amino acid positions 18 and 19 (Figure 3). Using ProtParam, the instability index of the complete preproform protein was computed to be 38.17 , which classifies the protein as stable. The deduced grand average of hydropathicity was -0.354 , demonstrating that this protein was hydrophobic. This result is the same as that predicted by the Hydrophobicityplot 1.0 software.
Subcloning, expression and Western blotting

After the recombinant plasmid pMD19-T-Der $f 1$ was digested by both $\mathrm{BamHI}$ and $\mathrm{Xhol}$, the recovered cDNA encoding Der $f 1$ was subcloned into the expression vector of $\mathrm{pET} 28 \mathrm{a}(+)$ and identified by restriction analysis. The plasmid pET28a(+)-Der $\mathrm{f} 1$ was then transformed into $E$. coli BL21 and the protein was expressed in the presence of the IPTG inducer. A single band of about $34 \mathrm{kDa}$ from SDSPAGE and Western blotting was observed, in accordance with prediction results for the sequence encoding Der $f 1$ without the sequence for 18 residues of the single peptide (Figure $4 \mathrm{~A}$ and $\mathrm{B}$ ).

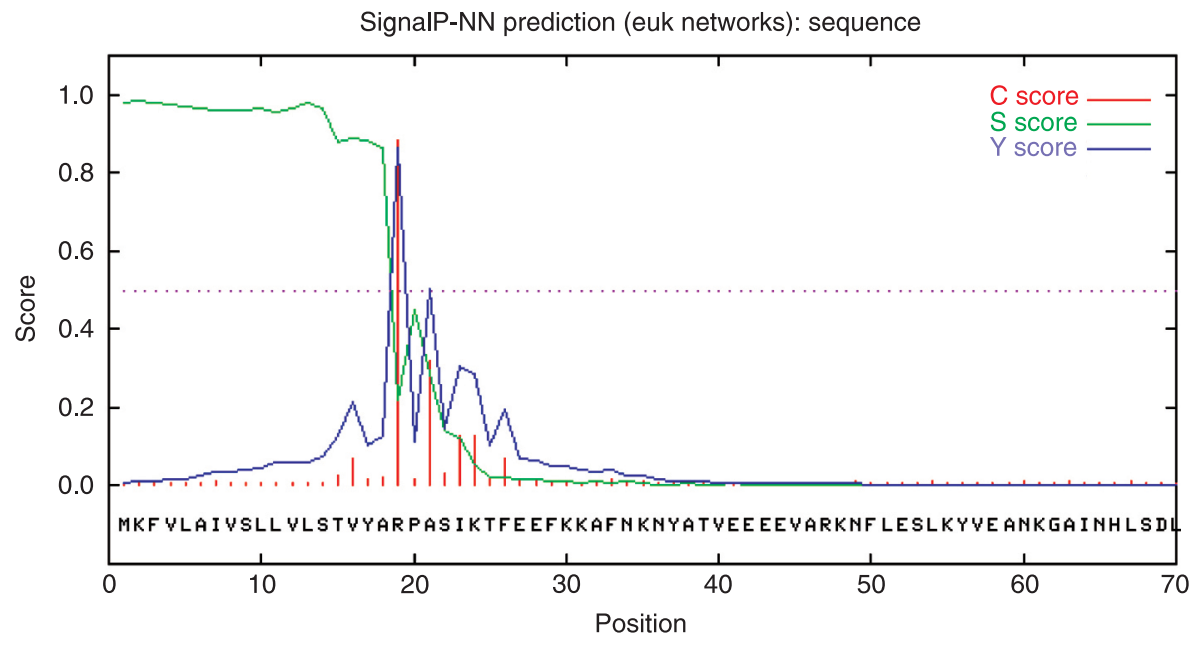

Figure 3. Prediction of signalPNN using the euk networks of the SignalP 3.0 software (http://www. cbs.dtu.dk/services/SignalP/). The most likely cleavage site between the positions 18 and 19 is VYA-RP.

A

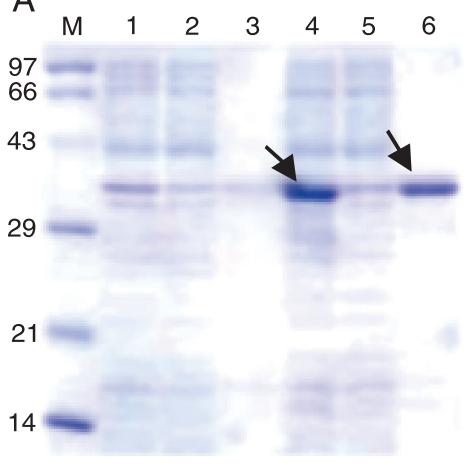

B

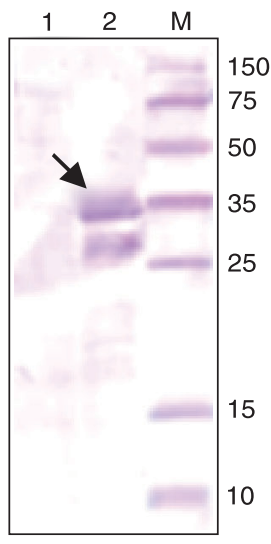

Figure 4. Expression rDer $\mathrm{f} 1$ in Escherichia coli BL21 cells. E. coli BL21 cells were transformed with either pET28a(+)-Der $f 1$ or empty vector pET28a(+) as control. SDS-PAGE (stained with CBB-R250) and Western blotting of rDer $f 1$ protein were performed as described in Material and Methods. A, SDS-PAGE analysis of $r$ Der $f 1$ protein; lane 1 , the whole cell lysate of $E$. coli BL21 cells containing pET28a; lane 2, the supernatant of cells containing pET28a; lane 3, the pellet of cells containing pET28a; lane 4 , the whole cell lysate of $E$. coli BL21 cells containing pET28a(+)-Der $f 1$; lane 5 , the supernatant of cells containing pET28a(+)-Der $f 1$; lane 6 , the pellet of cells containing $\mathrm{pET} 28 \mathrm{a}(+)$-Der $\mathrm{f} 1$. $B$, Western blotting of $\mathrm{rDer} \mathrm{f} 1$ protein; lane 1 , the whole cell lysate of $E$. coli BL21 cells containing pET28a; lane 2, the whole cell lysate of $E$. coli BL21 cells containing pET28a(+)-Der $f 1$. $M$ represents protein markers. The arrows point to rDer $f 1$ whose molecular mass is about $34 \mathrm{kDa}$. 
Blot

(1)

Sui m (1) --ASLNRDITFEQFKELYGKQYTAE-EEPQRRAIFEENLRWIQEN-----HGKHGAGLEVNEHADLTAEEFSSMYATLNQ

Boo m (1) ASSQEILRTQWEAFKTTHKKSYQSHMEELLRFKIFTENSLIIAKHNAKYAKGLVSYKLGMNQFGDLLAHEFARIFNGHHG

Sar s (1) --NPTEPIRTFKQFKETFGKSYANSFEETRAMKNFYESLAFVLR----_-----TNGTAINAHSDMSTEEFGRFFTMSER

Der p (1) --------------------------------NFLESVKYVQ-----------SNGGAINHLSDLSLDEFKNRFLMSAE

Der f (1) ---RPASIKTFEEFKKAFNKNYATVEEEEVARKNFLESLKYVE----_----ANKGAINHLSDLSLDEFKNRYLMSAE

Eur m (1) ---RPASIKTFEEFKKAFNKTYATPEKEEVARKNFLESLKYVE----_-----SNKGAINHLSDLSLDEFKNQFLMNAN 81

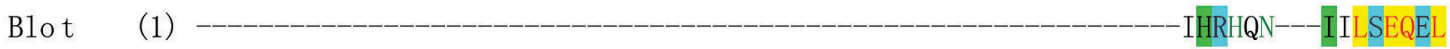

Sui m (73) EAFLKSPLHKEFVQVPESDIS-VALPAAFDWRQQ-WNTAVRNQGQCGSCWAFATAATVEAQYAIRKNVH---VTLSEQQL

Boo m (81) TRKTGGSTFLP-----PANVNDSSLPKVVDWRKKGAVTPVKDQGQCGSCWAFSATGSLEGQHFLKNGEL---VSLSEQNL

Sar s (69) QMKSIQEDYSL---I-ACRFNQTHFQSEIDLRKCGFVTPVKDQKKCGACWAFSTVCTTESLYLSSRQVSPWKFGLSEQEL

Der p (37) AFEHLKTQFDLNAETNACSIN-GNAPAEIDLRQMRTVTPIRMQGGCGSCWAFSGVAATESAYLAYRNQS---LDLAEQEL

Der f (67) AFEQLKTQFDLNAETSACRINSVNVPSELDLRSLRTVTPIRMQGGCGSCWAFSGVAATESAYLAYRNTS---LDLSEQEL

Eur m （67） AFEQLKTQFDLNAETYACSINSVSLPSELDLRSLRTVTPIRMQGGCGSCWAFSGVASTESAYLAYRNMS---LDLAEQEL 161

Blot (15) LDCTYHLYDPTYKCHGCQSGMSPEAFKYMKQ-KGLLEESHYPYKMKLNQCQANARG--TRYHVSSYNSLRYRAGDQEIQA

Sui m (148) VDCDHRPFQGQYEDHGCQGGNPIIAYAYVQQ-TGLVEESAYPYQARDGQCQSSTVNGHQRYHVSAGRELPFNATDETIMN

Boo m (153) VDCSQSFG-----NNGCEGGLMEDAFKYIKANDGIDTEKSYPYEAVDGECRFKKEDVG---ATDTGYVEIKAGSEVDLKK

Sar s (145) VDCAS--------PHGCDGDKMSVGFGYIEH-KGVGLSDQYPYIARVQPCQHCFGP---KFRIGGYCIIYPPDKTKIKVA

Der p (113) VDCAS--------QHGCHGDTIPRGIEYIQH-NGVVQESYYRYVAREQSCRRPNAQ---RFGISNYCQTYPPNANKIREA

Der f (144) VDCAS--------QHGCHGDTIPRGIEYIQQ-NGVVEERSYPYVAREQQCRRPNSQ---HYGISNYCQIYPPDVKQIREA

Eur m (144) VDCAS-------Q QNGCHGDTIPRGIEYIQQ-NGVVQEHYYPYVAREQSCHRPNAQ---RYGLKNYCQISPPDSNKIRQA 241

320

B1ot (92) AIMNHGPVVIYIHGTEAHFRNLRKG--ILRGAGYNDAQIDHAVVLVGWGTQNGIDYWIVRTSWGTQWGDAGYGFVERHHN

Sui m (227) SLHQIGPMAVLIFASDNEFRFYRNG--VIQNLRPNSRQINHAVTLVGWGTEDGQDYWIVKNSWGPSWGESGYFRLGRHHN

Boo m (225) AVATVGPISVAIDASHSSFQLYSEG--VYDEPECSSEDLDHGVLVVGYGVKGGKKYWVKNSWAESWGDQGYILMSRDNN

Sar s (213) MTVVQSAVSAVLLIEDLASFKHYDGKSVISSESKRSKTYGHGVNIVGYGSKYGQEVWIVRNSWGTTWGDKGYAYFAQNST

Der p (181) LAQTHSAIAVIIGIKDLDAFRHYDGRTIIQRDNGYQPNY-HAVNIVGYSNAQGVDYWIVRNSWDTNWGDNGYGYFAANID

Der f (212) LTQTHTAIAVIIGIKDLRAFQHYDGRT I IQHDNGYQPNY-HAVNIVGYGSTQGVDYWIVRNSWDTTWGDSGYGYFQAGNN

Eur m (212) LTQTHTAVAVIIGIKDLNAFRHYDGRTIMQHDNGYQPNY-HAVNIVGYGNTQGVDYWIVRNSWDTTWGDNGYGYFAANIN $321 \quad 335$

Blot (170) -SLGINNYPIYASL-

Sui m (305) -LIGINNYVFYPVL-

Boom (303) NQCGIASQASYPLV-

Sar s (293) -VMKLTKNVYMAWLH

Der p (260) -LMMIEEYPYVVIL-

Der f (291) -LMMIEQYPYVVIM-

Eur m （291） -LMMIEQYPYVVML-

Figure 5. Alignment of Der $\mathrm{f} 1$ with other group 1 allergens and cysteine proteases from ticks and mites determined using the VECTOR NTI 9.0 software. Group 1 allergens for Dermatophagoides farinae, Blomia tropicalis, D. pteronyssinus, Euroglyphus maynei, Sarcoptes scabiei, and Suidasia medanensis are abbreviated as Der f, Blo t, Der p, Eur m, Sar s, and Sui m, respectively. The tick Boophilus microplus is abbreviated as Boo $\mathrm{m}$. 
Table 1. Analysis of the similarity (\%) of Der $f 1$ and other group 1 allergens or cysteine proteases from ticks and mites using the VECTOR NTI 9.0 software.

\begin{tabular}{lccccccc}
\hline & Blo t & Sui $\mathrm{m}$ & Boo $\mathrm{m}$ & Sar s & Der $\mathrm{p}$ & Der $\mathrm{f}$ & Eur m \\
\hline Blo t & - & 29 & 18 & 20 & 32 & 24 & 23 \\
Sui m & 11 & - & 32 & 27 & 29 & 33 & 31 \\
Boo m & 12 & 20 & - & 27 & 24 & 28 & 28 \\
Sar s & 10 & 19 & 14 & - & 44 & 48 & 47 \\
Der $\mathrm{p}$ & 11 & 16 & 14 & 14 & - & 77 & 78 \\
Der $\mathrm{f}$ & 10 & 15 & 14 & 15 & 6 & - & 88 \\
Eur m & 10 & 17 & 13 & 15 & 7 & 6 & -
\end{tabular}

Der $\mathrm{f}$, Blo t, Der $\mathrm{p}$, Eur $\mathrm{m}$, Sar $\mathrm{s}$, and Sui $\mathrm{m}$ are group 1 allergens of Dermatophagoides farinae, Blomia tropicalis, D. pteronyssinus, Euroglyphus maynei, Sarcoptes scabiei, and Suidasia medanensis, respectively, and Boo $\mathrm{m}$ represents the tick Boophilus microplus. For sequence source information, see the section "Amino acid sequence homology analysis, alignment, and phylogeny".

Amino acid sequence homology analysis, alignment and phylogeny

The homology between the putative amino acid sequence of Der $f 1$ and other proteins was determined by comparing their sequences in all non-redundant GenBank CDS translations + PDB + SwissProt + PIR + PRF, excluding environmental samples, using BLASTp in the NCBI website. Based on our BLASTp search results, the Der $f 1$ amino sequences of ticks and mites, i.e., Blomia tropicalis (GenBank AAK58415), Boophilus microplus (GenBank AAF61565), D. pteronyssinus (GenBank ABA39435), E. maynei (GenBank P25780), Sarcoptes scabiei (GenBank AAS93667), and Suidasia medanensis (GenBank AAX34043) were chosen and, after sequences coding for signal peptides were deleted, they were aligned using the Vector NTI 9.0 software. A phylogenetic tree was then constructed using the Treeview 5.01.81 software. Interestingly, Der f 1 had $88 \%$ identity to Eur $\mathrm{m} 1$ in the amino acid sequence, while it had only $77 \%$ sequence identity to Der $\mathrm{p} 1$ (Figure 5 and Table 1). And $D$. farinae was clustered with $E$. maynei but not with $D$. pteronyssinus in the polygenic tree for their group 1 allergens (Figure 6).

Prediction of protein subcellular localization, specific motifs, secondary structure, and three-dimensional structures

Using CELLO ver. 2.5, it was concluded that the subcelIular localization of Der $f 1$ was extracellular (Table 2). Four different softwares (InterProScan, ScanProsite, PPSearch, and PROSITE) were used in the present study to predict the specific motifs of the Der $\mathrm{f} 1$ protein. Interestingly, all analyses showed the same results. There are three cysteine
Table 2. Prediction results for protein subcellular localization by CELLO version 2.5 (http://cello.life.nctu.edu.tw/).

\begin{tabular}{lcc}
\hline SVM & Localization & Reliability \\
\hline Amino acid composition & Extracellular & 0.528 \\
N-peptide composition & Extracellular & 0.517 \\
Partitioned sequence composition & Extracellular & 0.765 \\
Physicochemical composition & Cytoplasmic & 0.292 \\
Neighboring sequence composition & Extracellular & 0.741 \\
CELLO prediction & & \\
& Extracellular & $2.609^{*}$ \\
& Cytoplasmic & 0.743 \\
& Nuclear & 0.421 \\
& Lysosomal & 0.415 \\
& Mitochondrial & 0.173 \\
& Plasma membrane & 0.140 \\
& Vacuole & 0.131 \\
& Peroxisomal & 0.124 \\
& Chloroplast & 0.109 \\
& Endoplasmic reticulum & 0.093 \\
& Golgi & 0.023 \\
& Cytoskeletal & 0.021 \\
\hline
\end{tabular}

SVD = support vector machine

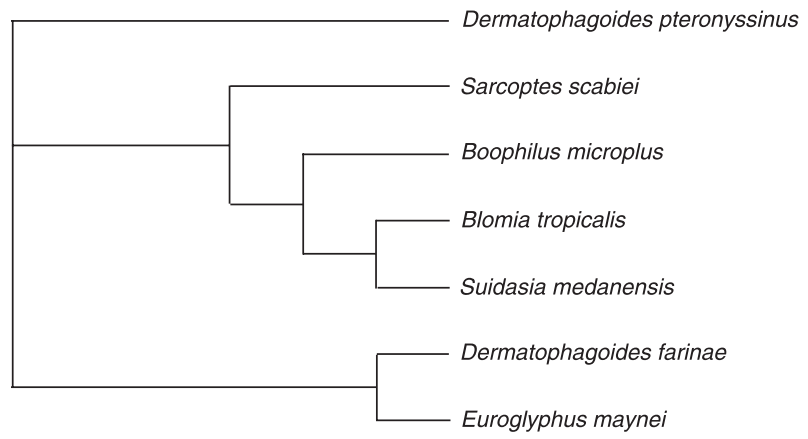

Figure 6. Phylogenetic tree constructed for Der $f 1$ and other group 1 allergens or cysteine proteases from ticks or mites using ClustalX 1.81.

peptidase active sites in this protein, respectively positioned at 127-138 (QGGCGSCWAFSG), 267-277 (NYHAVNIV GYG) and 284-303 (YWIVRNSWDTTWGDSGYGYF). The secondary structure of Der $f 1$ was predicted using the SOPMA software and the results showed that $33.96 \%$ of the protein (109 amino acids) was the $\alpha$ helix, $17.13 \%$ (55 amino acids) was the extended strand, $5.61 \%$ (18 amino acids) the ß turn, and $43.30 \%$ (139 amino acids) were random coils (Figure 7). In order to further understand the spatial arrangement of the three cysteine peptidase active sites in the threedimensional structure of this protein, we then imported the amino acid sequence into the Protein Data Bank (PDB, http:/ /www.rcsb.org/pdb/home/home.do) and found a template (ID: 1XKG). Subsequently, we submitted both our amino acid sequence and the template to the Swiss-model server 


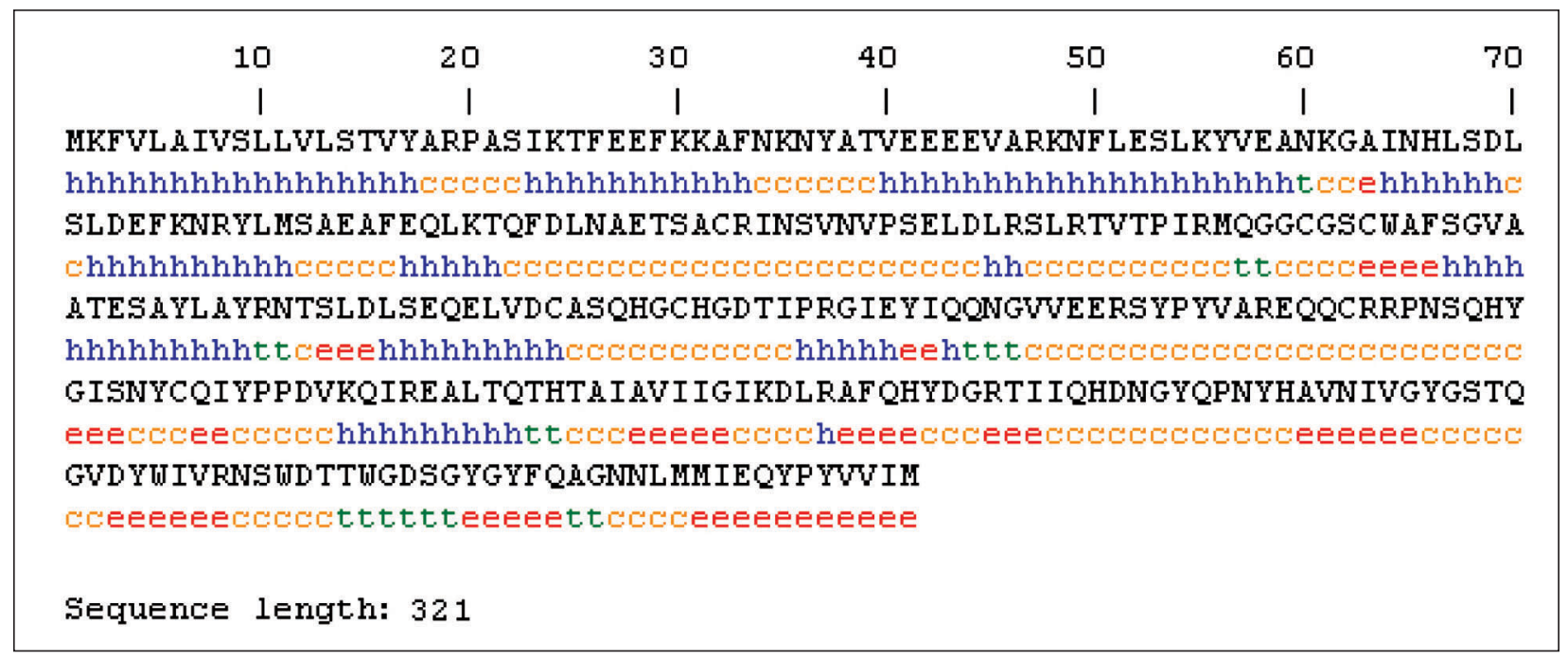

Figure 7. Secondary structure prediction of Der $f 1$ protein using the Sopma method (http://npsa-pbil.ibcp.fr/cgi-bin/ npsa_automat.pl?page=npsa_sopma.html). $\alpha$ helix (Hh); extended strand (Ee); $\beta$ turn (Tt); random coil (Cc).

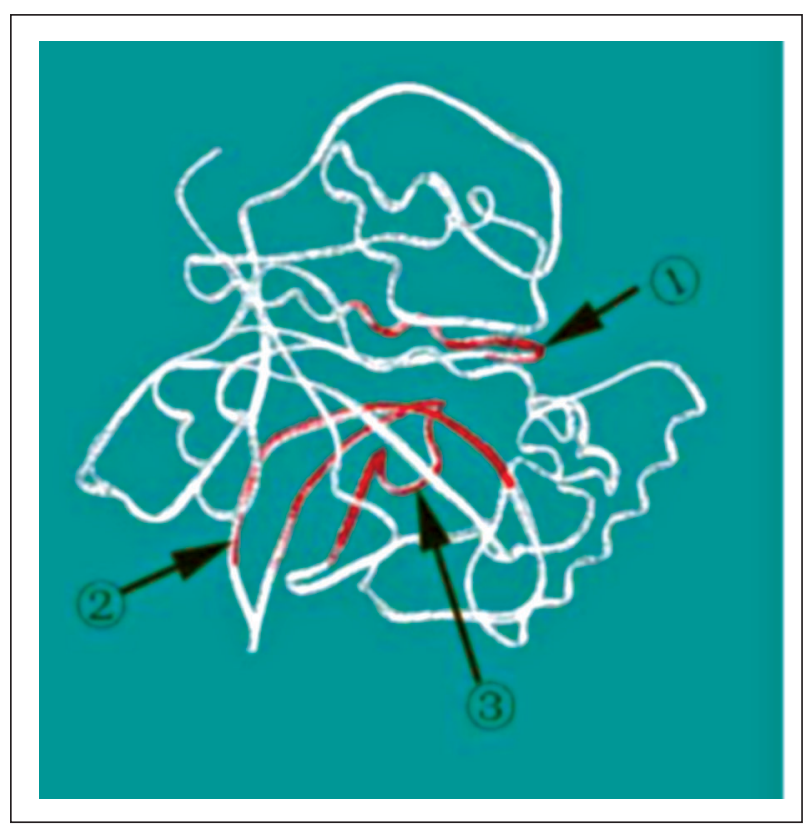

Figure 8. Three-dimensional model of the Der $\mathrm{f} 1$ protein constructed by Swiss-PdbViewer version 3.7. The three cysteine peptide active regions are colored red and indicated by the arrows: 1) residues 127-138 (QGGCGSCWAFSG), 2) residues 267-277 (NYHAVNIVGYG), and 3) residues 284-303 (YWIVRNSWDTTWGDSGYGYF).

(http://swissmodel.expasy.org), and obtained a simple threedimensional model of this protein in which we highlighted the three cysteine peptidase active sites using the Swiss-Pdb Viewer version 3.7 (Figure 8).

\section{Discussion}

A large body of evidence indicates that recombinant allergens are helpful to identify and produce the important IgE-binding determinants present on proteins which cause allergic reactions to house dust mites $(5,6)$. In the present study, a cDNA coding for the major house dust mite allergen Der $f 1$ was cloned and its DNA sequence was determined. A complete ORF was found in the cloned fragment with a full length of $966 \mathrm{bp}$. Sequence comparisons showed only $1 \mathrm{bp}$ difference between our fragment and that of the reference sequence (GenBank AB034946). Using the ProtParam Tools, the protein with 321 amino acids was found to have a molecular mass of $36,4189 \mathrm{kDa}$. However, this is only a theoretical prediction, since the sequence contained characteristic features of signal peptides with 18 amino acid residues at the 5 ' proximal end. In addition, the pro-peptides with 80 amino acid residues that have been reported previously (3) should be included. Once the presumptive pre- (54 bp) and pro-peptides (240 bp) were deleted, the remaining nucleotide sequence (672 bp) was predicted by the ProtParam Tools and the results showed a molecular mass of $25,148 \mathrm{kDa}$, the same as that reported earlier for Der $f 1$ sequence (3).

The active Der $\mathrm{f} 1$ protein with 223 residues has received a lot of attention and was cloned and expressed. In contrast, subsequent experiments demonstrated that Der $\mathrm{f} 1$ directly expressed in $E$. coli without the pro-sequence had very low IgE binding activity. For example, Best et al. (7) insisted that the pre- or pro-sequence ought to result in 
secretion of the mature form of the protein and that both the $\alpha$ factor signal peptide and the pro-enzyme region were efficiently processed during secretion, and the recombinant forms of Der $f 1$ ( $r$ Der $f 1$ ) expressed with pro-sequence in Pichia pastoris had similar IgE binding activity to native forms of Der $\mathrm{f} 1$ (nDer $\mathrm{f} 1$ ) obtained from mites. In the present study, we successfully expressed the allergen in E. coli BL21 with the complete preliminary sequence. Despite the absence of exact molecular weight data, we observed a single band of about $34 \mathrm{kDa}$ using SDS-PAGE and Western blotting, in accordance with predicted results of $34,469 \mathrm{kDa}$ with the sequence of the signal peptide (18 residues) being deleted.

Interestingly, sequence analyses suggest that $D$. farinae was evolutionarily more similar to $E$. maynei than to $D$. pteronyssinus, even though $D$. farinae and $D$. pteronyssinus are classified as belonging to the same Dermatophagoides genus. Firstly, Der $\mathrm{f} 1$ had $88 \%$ identity to Eur $\mathrm{m} 1$ in the amino acid sequence, while it had only $77 \%$ sequence identity to Der $p 1$. Secondly, $D$. farinae was clustered with $E$. maynei but not with $D$. pteronyssinus in the polygenic tree for their group 1 allergens. The results suggest either one of two hypotheses. The first is that the taxonomy for mites and ticks on the basis of morphology may not correctly reflect their true evolutionary history. Alternatively, the sequences of cysteine proteases may not correctly reflect the evolution of ticks and mites. More genes need to be analyzed to test these two hypotheses.

It is widely believed that the major dust mite allergen Der $f 1$ may be a digestive protease secreted by the gastrointestinal tract of mites $(1,2,8)$. Based on its biochemical and biophysical properties, the predicted subcellular localization of Der $\mathrm{f} 1$ was also extracellular, in agreement with previous observations. Our bioinformatic analyses identified three cysteine peptidase active sites and these site data were used to construct a simple threedimensional model of the molecule.

In conclusion, we successfully cloned, sequenced and expressed Der $\mathrm{f} 1$ in E. coli BL21, and identified some of its molecular characteristics using bioinformatic software. In particular, we drew the preliminary conclusion that $D$. pteronyssinus is evolutionarily more similar to $E$. maynei than to $D$. farinae, which will be confirmed using some molecular markers in the future.

\section{Acknowledgments}

We are grateful to Dr. Wang Bo, Faculty of Health Sciences, University of Cape Town, South Africa, to Dr. Xu Jianping, Department of Biology, McMaster University, Canada, and to Dr. M. Medeiros Jr., Serviço de Imunologia, Hospital Universitário Prof. Edgar Santos, Salvador, $\mathrm{BA}$, Brazil, for their patient revision of our English writing.

\section{References}

1. Thomas WR, Smith WA, Hales BJ. The allergenic specificities of the house dust mite. Chang Gung Med J 2004; 27: 563-569.

2. Thomas WR, Smith WA, Hales BJ, Mills KL, O'Brien RM. Characterization and immunobiology of house dust mite allergens. Int Arch Allergy Immunol 2002; 129: 1-18.

3. Dilworth RJ, Chua KY, Thomas WR. Sequence analysis of cDNA coding for a major house dust mite allergen, Der $\mathrm{f}$ I. Clin Exp Allergy 1991; 21: 25-32.

4. Cui YB, Ling YZ, Zhou Y, Feng ZW, Xing YR, Zhang SW. An effective indirect fluorescent antibody test for diagnosis of intestinal acariasis. Southeast Asian J Trop Med Public Health 2006; 37: 452-455.

5. Smith W, Mills K, Hazell L, Hart B, Thomas W. Molecular analysis of the group 1 and 2 allergens from the house dust mite, Euroglyphus maynei. Int Arch Allergy Immunol 1999; 118: $15-22$.

6. Mora C, Flores I, Montealegre F, Diaz A. Cloning and expression of Blo $t$, a novel allergen from the dust mite Blomia tropicalis, homologous to cysteine proteases. Clin Exp Allergy 2003; 33: 28-34.

7. Best EA, Stedman KE, Bozic CM, Hunter SW, Vailes L, Chapman MD, et al. A recombinant group 1 house dust mite allergen, $r$ Der $\mathrm{f} 1$, with biological activities similar to those of the native allergen. Protein Expr Purif 2000; 20: 462-471.

8. Tovey ER, Chapman MD, Platts-Mills TA. Mite faeces are a major source of house dust allergens. Nature 1981; 289: 592-593. 